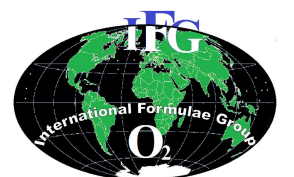

Available online at http://ajol.info/index.php/ijbcs

Int. J. Biol. Chem. Sci. 8(6): 2866-2878, December 2014

ISSN 1997-342X (Online), ISSN 1991-8631 (Print)
International Joumal

of Biological and

Chemical Sciences

Original Paper

http://indexmedicus.afro.who.int

\title{
Le phosphore limite-t-il la production intensive du riz dans la plaine de Bagré au Burkina Faso?
}

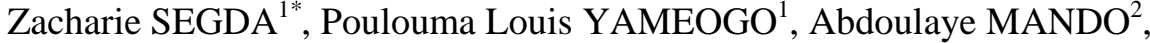 \\ Saito KAZUKI ${ }^{3}$, Marco C.S. WOPEREIS ${ }^{3}$ et Michel Papaoba SEDOGO ${ }^{1}$ \\ ${ }^{1}$ Institut de l'Environnement et de Recherches Agricoles (INERA), \\ 04 BP 8645, Ouagadougou 04, Burkina Faso. \\ ${ }^{2}$ Centre International pour la Fertilité des sols et le Développement Agricole (IFDC) - Africa Division, \\ BP 4483, Lomé, Togo. \\ ${ }^{3}$ Centre du Riz pour l'Afrique (AfricaRice), C/O IITA, 01 BP 2031 Cotonou, Bénin. \\ *Auteur correspondant, E-mail : zacharie.segda@yahoo.fr, Tél : +226 70270400
}

\section{RESUME}

Le phosphore $(\mathrm{P})$ est un élément important dans la nutrition minérale du riz et dans l'élaboration du rendement paddy. Des essais soustractifs ont été conduits en milieu paysan dans la plaine rizicole de Bagré au cours de trois campagnes successives pour évaluer l'effet de l'application de $\mathrm{P}$ sur le rendement paddy. La capacité nutritive du sol en phosphore a varié entre 7 et $15 \mathrm{~kg} \mathrm{P} \mathrm{ha}^{-1}$. L'application de $\mathrm{P}$ a augmenté l'efficacité

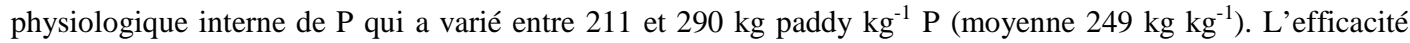
agronomique de $\mathrm{P}$ a été de $33,7 \mathrm{~kg}$ paddy $\mathrm{kg}^{-1}$ de $\mathrm{P}$ appliqué. L'augmentation de rendement due à l'application de phosphore a été de 19\%. En moyenne, $70 \%$ du phosphore total absorbé est contenu dans les grains. L'évolution du rendement paddy indique que $\mathrm{P}$ est le second facteur limitant dans la plaine de Bagré après l'azote. L'application de $\mathrm{P} \_$engrais à une dose moyenne de $30 \mathrm{~kg} \mathrm{P} \mathrm{ha}^{-1}$ était suffisante pour compenser les pertes dues aux exportations. Le phosphore limite la production de riz à Bagré mais son application comme engrais contribue à améliorer les rendements paddy et à compenser les pertes de $\mathrm{P}$ du système.

(C) 2014 International Formulae Group. All rights reserved.

Mots clés : Capacité nutritive des sols, rizières, efficacité d'utilisation de nutriments, bilan minéral.

\section{INTRODUCTION}

Les cultures irriguées et principalement le riz constituent de plus en plus un élément stratégique de la recherche de la sécurité alimentaire du Burkina Faso. Cette stratégie est présentement développée par l'aménagement et la mise en valeur de nouveaux bas-fonds et périmètres comme celui de Bagré, mais aussi par l'intensification de la riziculture irriguée. Le riz irrigué occupe seulement $23 \%$ des superficies emblavées en riz sur la période 1984-2009 mais sa contribution dans la production nationale de riz commercialisé atteint plus de 53\% (INERA-DGPER, 2010). Cette contribution du riz irrigué bien qu'en croissance, peut être fortement améliorée car les rendements réalisés sont encore très en dessous des rendements potentiels ou réalisables (Wopereis et al., 2001; De Vries et al., 2011). En effet, le potentiel de production du riz irrigué (production seulement limitée par le 
climat et les performances de la variété utilisée) au Burkina Faso peut atteindre 8-10 t $\mathrm{ha}^{-1}$, mais les rendements moyens actuels sont de $4 \mathrm{t} \mathrm{ha}^{-1}$ (Segda, 2001), soit moins de 50\% du potentiel de la zone et de celui des variétés de riz cultivées (De Vries et al., 2011). Ces écarts de rendements qui concernent toute la zone soudano sahélienne (van Asten et al., 2003) sont surtout liés à une mauvaise couverture des besoins des plantes en éléments minéraux du sol, particulièrement le phosphore, l'azote et le potassium. Ensuite, l'action des ennemis des cultures et la qualité de gestion de la rizière comme la préparation $\mathrm{du}$ sol, la gestion des mauvaises herbes déterminent aussi les performances du riz (Wopereis et al., 2001; van Asten et al., 2003). Enfin, le non-respect du calendrier cultural contribue fortement à l'augmentation des écarts entre le rendement potentiel et le rendement moyen actuel (Wopereis et al., 2001; FAO, 2003).

Les recommandations actuelles de fertilisation du riz irrigué à Bagré et plus généralement au Burkina Faso sont surtout basées sur les besoins en éléments nutritifs des cultures, sans que le stock d'éléments nutritifs dans le sol soit pris en considération (Segda, 2001). Apprécier cette "capacité du sol à fournir des nutriments" est d'un grand intérêt puisque cela permet de mieux adapter les apports d'engrais minéraux aux besoins en nutriments des cultures. En effet, ignorer les réserves disponibles des sols en éléments nutritifs peut aboutir à des applications d'engrais qui ne coïncident pas avec les besoins des plantes en éléments nutritifs, d'où un gaspillage de nutriments, d'argent et même une pollution de l'environnement (Wang et al., 2001). L'apport d'engrais minéral doit prendre en considération la réserve en éléments nutritifs du sol. Les paysans s'en rendent compte, mais l'environnement institutionnel dans lequel ils opèrent ne laisse souvent guère de flexibilité. Dans la zone soudano sahélienne, l'urée $\left(46 \mathrm{~N}-0 \mathrm{P}_{2} \mathrm{O}_{5}-\mathrm{K}_{2} \mathrm{O}\right)$ et le NPK $\left(14 \mathrm{~N}-23 \mathrm{P}_{2} \mathrm{O}_{5}-14 \mathrm{~K}_{2} \mathrm{O}\right)$ sont souvent les seuls engrais minéraux disponibles, en dehors de l'engrais composé spécifique pour le coton (Haefele, 2001; Haefele et al., 2002).
Certains éléments minéraux sont dans le sol à des niveaux insuffisants pour pourvoir à l'alimentation optimale des cultures. C'est premièrement le cas du phosphore ensuite de l'azote à cause des faibles teneurs en matière organique des sols, puis le soufre et dans une moindre mesure le potassium dont la déficience n'apparaît qu'au bout de cinq ou sept années de cultures successives (Haefele, 2001). L'effet du phosphore n'est pas visible comme celui de l'azote. Cependant, il permet une meilleure croissance racinaire, favorise un tallage plus actif, avec des talles fertiles et agit sur le bon développement des grains en élevant leur valeur alimentaire (Lacharme, 2001). La disponibilité du phosphore pour les plantes est plus faible dans les sols acides. L'application fractionnée n'est pas rentable. La production d'une tonne de riz paddy exige environ $20 \mathrm{~kg} \mathrm{~N}, 11 \mathrm{~kg} \mathrm{P}_{2} \mathrm{O}_{5}, 30 \mathrm{~kg} \mathrm{~K}_{2} \mathrm{O}$ (Roy et al., 2006). Sur la consommation totale de la plante de riz, $50 \%$ d'azote, $50 \%$ de potassium et $65 \%$ de phosphore sont pris dès le début de l'initiation paniculaire. Environ $80 \%$ d'azote, $60 \%$ de potassium et $95 \%$ de phosphore sont absorbés pendant l'épiaison. C'est pourquoi $\mathrm{P}$ est en général appliqué comme fumure de fond au moment du semis/repiquage.

L'objectif principal de l'étude était de déterminer l'importance du phosphore dans la production intensive de riz irrigué sur la plaine de Bagré au Burkina Faso. Il s'agissait plus spécifiquement de déterminer la capacité nutritive des sols en phosphore, d'évaluer le rendement $\mathrm{du}$ riz avec l'application $\mathrm{du}$ phosphore, d'estimer l'efficacité d'utilisation de P-engrais apporté et d'établir un bilan minéral partiel du phosphore dans les conditions agro-pédo-climatiques de Bagré.

\section{MATERIEL ET METHODES Site d'étude}

L'étude a été conduite dans le périmètre irrigué de Bagré situé dans le Centre Est du Burkina Faso, sur le Plateau Central (11 $\left.{ }^{\circ} 27^{\prime} 04^{\prime \prime} \mathrm{N} ; 0^{\circ} 28^{\prime} 23^{\prime \prime} \mathrm{O}\right)$. Le climat est caractéristique de la zone agro-écologique de savane soudano-sahélienne (BEGE, 2008). Les sols du périmètre irrigué de Bagré sont issus de formations alluviales du Quaternaire. 
Il s'agit de Gleysols district Fluvisols, avec une profondeur se situant entre 0,4 et 1,2 m.

\section{Matériel}

Les producteurs pratiquant le système de double culture annuelle de riz en hivernage (HIV) et en contre saison sèche chaude (CSC) ont été concernés par les essais. Les variétés utilisées ont été choisies par les producteurs, en prenant soin que ce soit la même variété dans tous les traitements de chaque champ. Seules ont été utilisées les variétés modernes à haut potentiel de rendement avec un index de récolte situé entre 0,40 et 0,50 (par exemple TOX 7281, 4418, TS2, ou TCS10). La densité de repiquage de $0,20 \mathrm{~m} \times 0,20 \mathrm{~m}$ avec en moyenne 36 touffes au $\mathrm{m}^{2}$ était la forme principale d'établissement des cultures à Bagré en hivernage comme en contre saison sèche chaude (Segda, 2001). La gestion de l'eau et le contrôle des ravageurs par les producteurs sont effectués selon le protocole recommandé. Là où des problèmes sont suspectés ou observés, des mesures de contrôle prophylactiques ou curatives sont appliquées sous la supervision des chercheurs. Le principe de base est de minimiser autant que possible les effets négatifs des facteurs autres que les nutriments pour maximiser les performances des itinéraires techniques appliqués.

\section{Méthodes}

\section{Dispositif expérimental}

Des essais soustractifs ont été conduits en milieu paysan à Bagré, pendant deux campagnes humides successives (2003 et 2004) et une campagne sèche (2004). Le dispositif expérimental comprenait cinq traitements :

- 0 NOPOK, témoin absolu sans fertilisation [0 $\left.\mathrm{kg} \mathrm{ha}^{-1} \mathrm{~N}, 0 \mathrm{~kg} \mathrm{ha}^{-1} \mathrm{P}, 0 \mathrm{~kg} \mathrm{ha}^{-1} \mathrm{~K}\right]$.

- $0 \mathrm{~N}$, traitement sans $\mathrm{N}$, mais avec $\mathrm{P}$ et $\mathrm{K}[0$ $\left.\mathrm{kg} \mathrm{ha}^{-1} \mathrm{~N}, 30 \mathrm{~kg} \mathrm{ha}^{-1} \mathrm{P}, 35 \mathrm{~kg} \mathrm{ha}^{-1} \mathrm{~K}\right]$.

- $0 \mathrm{P}$, traitement sans $\mathrm{P}$, mais avec $\mathrm{N}$ et $\mathrm{K}$ [120 kg ha $\left.{ }^{-1} \mathrm{~N}, 0 \mathrm{~kg} \mathrm{ha}{ }^{-1} \mathrm{P}, 35 \mathrm{~kg} \mathrm{ha}^{-1} \mathrm{~K}\right]$.

- $0 \mathrm{~K}$, traitement sans $\mathrm{K}$, mais avec $\mathrm{N}$ et $\mathrm{P}$ [120 kg ha $\left.{ }^{-1} \mathrm{~N}, 30 \mathrm{~kg} \mathrm{ha}^{-1} \mathrm{P}, 0 \mathrm{~kg} \mathrm{ha}^{-1} \mathrm{~K}\right]$.
- NPK, traitement avec N, P et K $\left[120 \mathrm{~kg} \mathrm{ha}^{-1}\right.$ $\left.\mathrm{N}, 30 \mathrm{~kg} \mathrm{ha}^{-1} \mathrm{P}, 35 \mathrm{~kg} \mathrm{ha}^{-1} \mathrm{~K}\right]$.

Les parcelles diagnostiques de $5 \mathrm{~m} \times 5$ $\mathrm{m}\left(25 \mathrm{~m}^{2}\right)$ ont été établies dans chaque champ paysan test et séparées du reste par des diguettes afin d'éviter les contaminations éventuelles lorsque le paysan applique l'engrais dans ses parcelles en cours de saison. Les parcelles diagnostiques sont implantées dans le même champ que la campagne suivante mais en des emplacements différents afin de représenter les conditions normales de la capacité nutritive du sol sous gestion normale des cultures, d'éviter l'épuisement des nutriments et de disposer d'estimations sur la capacité nutritive des sols dans le temps. Chaque producteur a utilisé la même variété (soit TOX 728-1, 4418, TS2, ou TCS10) au cours des trois cycles culturaux.

L'azote a été apporté sous forme d'urée $(46 \% \mathrm{~N})$ à la dose de $120 \mathrm{~kg} \mathrm{~N} \mathrm{ha}{ }^{-1}$, le phosphore sous forme de triple super phosphate $\left(46 \% \mathrm{P}_{2} \mathrm{O}_{5}\right)$ à la dose de $30 \mathrm{~kg} \mathrm{P}$ $\mathrm{ha}^{-1}$ et le potassium sous forme de chlorure de potassium $\left(60 \% \mathrm{~K}_{2} \mathrm{O}\right)$ à la dose de $35 \mathrm{~kg} \mathrm{~K}$ $\mathrm{ha}^{-1}$. Le potassium $(\mathrm{K})$ a été apporté en fond (avant le repiquage), dans les traitements "OP", "ON" et "NPK". Le phosphore (P) a été apporté 3 jours après repiquage (JAR) dans les traitements " $0 \mathrm{~K}$ ", "0N" et "NPK". La première fraction d'urée $(35 \%)$ a été apportée 18 JAR dans les traitements "OK", "OP" et "NPK" et la deuxième application d'urée $(65 \%)$ à l'initiation paniculaire dans les mêmes parcelles.

Treize producteurs ont conduit les tests en saison humide 2003, 17 en saison sèche 2004 et 12 en saison humide 2004.

\section{Capacité nutritive du sol}

La capacité nutritive du sol est estimée sur la base des essais soustractifs de $\mathrm{P}$ et des facteurs empiriques pour $\mathrm{P}$, en utilisant le facteur de correction du phosphore qui est de $2,9 \mathrm{~kg} \mathrm{P}$ absorbé par tonne de paddy (Dobermann et al., 2003a).

\section{Efficacité d'utilisation du phosphore}

Les paramètres qui ont été utilisés pour l'estimation de l'efficacité d'utilisation du phosphore sont l'efficacité agronomique de 
P_engrais apporté (EaP) et l'efficacité interne de $\mathrm{P} \_$engrais (EiP). L'efficacité agronomique

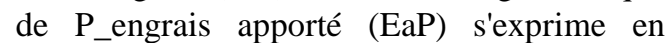
kilogramme de rendement paddy additionnel par kilogramme de phosphore appliqué :

$\mathrm{EaP}=(\mathrm{YP}-\mathrm{Y} 0) / \mathrm{FP}$

$\mathrm{FP}=$ quantité d'engrais $\mathrm{P}$ apportée (en $\mathrm{kg} \mathrm{P}$ $\mathrm{ha}^{-1}$ )

$\mathrm{YP}=$ rendement paddy avec l'application de $\mathrm{P}$ $\left(\right.$ en $\left.\mathrm{kg} \mathrm{ha}^{-1}\right)$

$\mathrm{Y} 0=$ rendement paddy $\left(\mathrm{en} \mathrm{kg} \mathrm{ha}^{-1}\right)$ dans des traitements sans application de $\mathrm{P}$

L'efficacité interne de $P_{\_}$engrais apporté (EiP) qui représente le rendement paddy par unité de phosphore absorbé s'exprime en $\mathrm{kg}$ paddy $\mathrm{kg}^{-1}$ d'élément nutritif P absorbé (Witt, 2003) :

EiP ( $k$ gaddy $\mathrm{kg}^{-1} \mathrm{P}$ absorbé $)=\mathrm{Y} / \mathrm{P}$ absorbé $\mathrm{Y}=$ rendement paddy obtenu avec application de P (en kg ha ${ }^{-1}$ )

$\mathrm{P}$ absorbé $=\mathrm{P}$ absorbé à maturité sur parcelles fertilisées avec $\mathrm{P}$ (en $\mathrm{kg} \mathrm{P}^{-1}$ )

EiP inclut l'élément $\mathrm{P}$ prélevé de $\mathrm{P} \_$engrais minéral apporté et celui provenant de la minéralisation du sol (P_sol). C'est donc une mesure globale du statut nutritionnel.

\section{Analyses de sols et de plantes}

Les prélèvements des échantillons de sols ont été effectués dans chaque parcelle paysanne à l'aide d'une tarière à la profondeur de 0-0,20 m, avant l'installation des cultures en juin 2003, en janvier 2004 et en juin 2004. Pour tenir compte de l'hétérogénéité du milieu, un échantillon composite constitué d'un mélange de cinq prélèvements (un dans chaque coin à $1 \mathrm{~m}$ au moins du bord et un au centre) a été fait. L'échantillon de sol a été séché à l'air puis passé au tamis à mailles de 2 $\mathrm{mm}$ et conservé dans des sachets plastiques. Les analyses chimiques ont concerné les échantillons prélevés en juin 2003, en janvier 2004 et en juin 2004. Le pH_eau a été mesuré par la méthode électrométrique au pH-mètre, après agitation pendant 30 minutes d'un mélange de $20 \mathrm{~g}$ de sol et de $50 \mathrm{ml}$ d'eau distillée. Les rapports sols-solution étaient donc de 1:2,5. Pour la détermination du $\mathrm{P}$ total, les échantillons de sol ont d'abord été minéralisés à chaud avec un mélange $\mathrm{H}_{2} \mathrm{SO}_{4}$ $\mathrm{Se}-\mathrm{H}_{2} \mathrm{O}_{2}$. Par la suite, les teneurs en $\mathrm{P}$ total ont été déterminées dans les minéralisâts, à l'aide d'un colorimètre automatique SKALAR (Segmented flow analyser, model SANplus 4000-02, Skalar Hollande). Le dosage du phosphore assimilable a été fait avec la méthode Olsen (Sims, 2000).

Les prélèvements d'échantillons de plantes ont été effectués suivant la procédure standard (Dobermann and Fairhurst, 2000). Pour l'estimation du rendement paddy, le riz a été récolté sur une surface de $6 \mathrm{~m}^{2}$ (3 $\mathrm{m}$ x $\left.2 \mathrm{~m}\right)$ dans chaque parcelle élémentaire. Les touffes ont été coupées à $10 \mathrm{~cm}$ au-dessus du sol. L'humidité des grains paddy est déterminée avec l'appareil de mesure "Riceter grain moisturemeter, Kett Electric Laboratory, Tokyo, Japan". Les rendements grains sont ramenés à $14 \%$ d'humidité. La concentration en phosphore des grains et de la paille a été déterminée à partir du prélèvement de 12 touffes. Les échantillons ont ensuite été séchés à l'étuve à $70{ }^{\circ} \mathrm{C}$ jusqu'à humidité constante de $3 \%$. Les analyses chimiques ont porté sur le phosphore total absorbé à maturité par le riz (grains et paille) selon la procédure décrite par van Reeuwijk (2002).

\section{Estimation du bilan partiel du phosphore}

Pour l'estimation du bilan minéral en phosphore, l'approche simplifiée qui permet le calcul du "bilan partiel" (Kanté, 2001; FAO, 2003) a été utilisée. Dans notre cas, le "bilan partiel" fait intervenir seulement comme «input», les apports de phosphore par les engrais minéraux et comme «outpout», les exportations de phosphore par les produits récoltés, notamment les grains et la paille.

\section{Analyses statistiques}

Les données collectées ont été soumises à une analyse de variance (ANOVA) en utilisant le logiciel STATITCF. Le test de Newman Keuls a été utilisé pour la séparation des moyennes au seuil de probabilité de $5 \%$.

\section{RESULTATS}

Analyse de sols et capacité nutritive des sols en phosphore

Pour les échantillons de sols qui ont été prélevés (Tableau 1), les valeurs du pH_eau ont été comprises entre 5,54 et 7,94. Les valeurs du P total ont été comprises entre 53 et $461 \mathrm{mg} \mathrm{kg}^{-1}$ sol et celles du phosphore assimilable entre 0,43 et $13,74 \mathrm{mg} \mathrm{kg}^{-1}$ sol. La 
capacité nutritive du sol en phosphore a été comprise entre 7 et $15 \mathrm{~kg} \mathrm{P}$ ha $^{-1}$ avec une moyenne de $11 \mathrm{~kg} \mathrm{P}^{-1}$ (Tableau 1).

Réponse du riz à l'application du phosphore et efficacité d'utilisation de $\mathbf{P}$ Réponse du riz à l'application de phosphore

La réponse du riz à l'application de phosphore est présentée dans la Figure 1. Pour toutes les campagnes rizicoles réunies et même pour chaque campagne rizicole (données non présentées), l'analyse de variance a montré une différence très hautement significative $(\mathrm{P}<0,0001)$ entre les différents traitements. Les rendements grains ont augmenté dans l'ordre 0NOP0K (3 $200 \mathrm{~kg}$ $\left.\mathrm{ha}^{-1}\right)<0 \mathrm{~N}\left(3918 \mathrm{~kg} \mathrm{ha}^{-1}\right)<$ OP (4 $\left.555 \mathrm{~kg} \mathrm{ha}^{-1}\right)$ $<$ 0K (4 $736 \mathrm{~kg} \mathrm{ha}^{-1}$ ) < NPK (5 $694 \mathrm{~kg} \mathrm{ha}^{-1}$ ). Les traitements qui ont fait ressortir l'apport réel de $\mathrm{P}$ dans la nutrition minérale du riz, à savoir NPK (apport complet des trois éléments nutritifs de base) et $0 \mathrm{P}$ (sans apport de $\mathrm{P}$, mais avec $\mathrm{N}$ et $\mathrm{K}$ ) ont été utilisés (Tableau 2). L'augmentation moyenne de rendement due à l'application de $\mathrm{P}$ pour les trois campagnes rizicoles a été de 19\%, augmentation de rendement qui s'est situé entre 14 et $25 \%$ en fonction de la saison de culture. Elle a été plus élevée en HIV 2004 (25\%).

Les rendements paddy dans les parcelles $0 \mathrm{~N}, 0 \mathrm{P}$ et $0 \mathrm{~K}$ ont été fortement corrélés entre eux (Figure $2 \mathrm{a}$ et $2 \mathrm{~b}$ ). La corrélation entre les rendements grains dans les traitements $0 \mathrm{~K}$ et les traitements $0 \mathrm{~N}$ a été forte $\left(r^{2}=0,61\right.$, Figure 3a). La plus forte corrélation a été trouvée entre les rendements grains dans les traitements $0 \mathrm{~N}$ et les traitements $\mathrm{OP}\left(\mathrm{r}^{2}=0,73\right.$, Figure $\left.3 \mathrm{~b}\right)$.

\section{Efficacité d'utilisation du phosphore}

L'efficacité agronomique de $\mathrm{P}(\mathrm{EaP}) \mathrm{a}$ varié de 22,2 à $39,7 \mathrm{~kg}$ paddy $\mathrm{kg}^{-1}$ de $\mathrm{P}$ appliqué obtenu en HIV 2003 et HIV 2004 (Tableau 2). L'EaP moyenne de l'ensemble des saisons a été de $33,7 \mathrm{~kg}$ paddy $\mathrm{kg}^{-1}$ de $\mathrm{P}$ appliqué. La différence entre les valeurs du phosphore assimilable du traitement NPK et le traitement témoin a été en moyenne de 0,042 $\mathrm{mg} \mathrm{P} \mathrm{kg}{ }^{-1}$ sol, variant entre 0,03 et $0,07 \mathrm{mg} \mathrm{P}$ $\mathrm{kg}^{-1}$ sol. L'efficacité interne de $\mathrm{P}$ (EiP) a été plus élevée en HIV 2003 (290 kg kg-1 de P absorbé). En l'absence d'apport de $\mathrm{P}$, l'efficacité interne de $\mathrm{P}$ (rendement paddy/P absorbé à maturité dans les parties aériennes) a varié entre 297 et $343 \mathrm{~kg}$ paddy $\mathrm{kg}^{-1} \mathrm{P}$ dans le traitement 0 NOPOK (moyenne $311 \mathrm{~kg} \mathrm{~kg}^{-1}$ $\left.\mathrm{P}, \mathrm{r}^{2}=0,32\right)$ et 294 et $1543 \mathrm{~kg}$ paddy $\mathrm{kg} \mathrm{P}^{-1}$ dans les traitements OP (moyenne $488 \mathrm{~kg} \mathrm{~kg}^{-1}$ $\mathrm{P}, \mathrm{r}^{2}=0,21$ ), fonction d'une faible relation entre le rendement paddy et le phosphore absorbé à maturité (Figure 3a et 3b). L'apport de $\mathrm{P}$ a amélioré très sensiblement l'efficacité interne de $\mathrm{P}$ qui a varié de 211 à $290 \mathrm{~kg}$ paddy $\mathrm{kg}^{-1} \mathrm{P}$ dans les traitements NPK (moyenne $249 \mathrm{~kg} \mathrm{~kg}^{-1} \mathrm{P}$ ), avec un $\mathrm{r}^{2}$ de 0,78 (Figure $3 \mathrm{c}$ ).

La concentration en $\mathrm{P}$ dans les grains et dans la paille est présentée dans le Tableau 3. La concentration en $\mathrm{P}$ a varié de 0,24 à $0,32 \%$ dans les grains (moyenne 0,28\%) et de 0,08 à 0,13 dans la paille (moyenne $0,10 \%$ ). On a observé des valeurs plus faibles dans les traitements «0NOP0K» et «0P» en particulier. En moyenne, $70 \%$ du phosphore total absorbé était contenu dans les grains.

La Figure 4 présente les relations entre le phosphore assimilable et le rendement paddy et entre le phosphore assimilable et l'accumulation de $\mathrm{P}$ à la maturité dans les essais soustractifs. Il n'y a pas eu de relation claire entre le rendement paddy dans les traitements sans phosphore et le phosphore assimilable (Figure 4a). De même, il n'y a pas eu de relation claire entre le phosphore absorbé à maturité $\left(\mathrm{r}^{2}=0,0019\right)$ et le phosphore assimilable (Figure 4b).

\section{Bilan partiel du phosphore $(\mathbf{P})$}

Le bilan partiel du phosphore (Tableau 4) a été négatif pour le traitement témoin (de 7 à $-11 \mathrm{~kg} \mathrm{P} \mathrm{ha}^{-1}$ ) et le traitement apportant l'azote et le potassium (de -8 à $-10 \mathrm{~kg} \mathrm{P} \mathrm{ha}^{-1}$ ), c'est-à-dire sans apport de phosphore. Par contre le bilan a été positif pour le traitement apportant les trois éléments nutritifs majeurs $\left(\mathrm{de}+6\right.$ à $\left.+14 \mathrm{~kg} \mathrm{P} \mathrm{ha}^{-1}\right)$. Lorsqu'on a apporté du phosphore (ensemble des trois campagnes rizicoles), le bilan moyen de $\mathrm{P}$ a été positif, allant de $+9 \mathrm{~kg} \mathrm{P} \mathrm{ha}^{-1}$ pour le traitement NPK, $+12 \mathrm{~kg} \mathrm{P} \mathrm{ha}^{-1}$ pour $0 \mathrm{~K}$, à $+16 \mathrm{kgP} \mathrm{ha}^{-1}$ pour le traitement $0 \mathrm{~N}$. Sans apport de P, le bilan moyen a été négatif, les valeurs observées ont été de $-9 \mathrm{~kg} \mathrm{P} \mathrm{ha}^{-1}$ pour le témoin et $\mathrm{OP}$. 


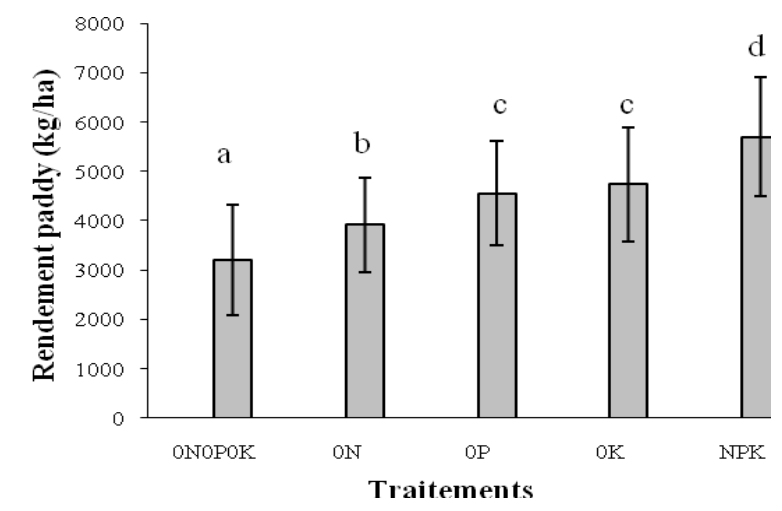

Figure 1: Effet des éléments nutritifs appliqués sur le rendement du riz irrigué à Bagré pour l'ensemble des trois campagnes rizicoles. Moyenne de l'hivernage 2003, de la contre saison sèche 2004 et de l'hivernage 2004 ; Les barres représentent l'écart type par rapport à la moyenne.
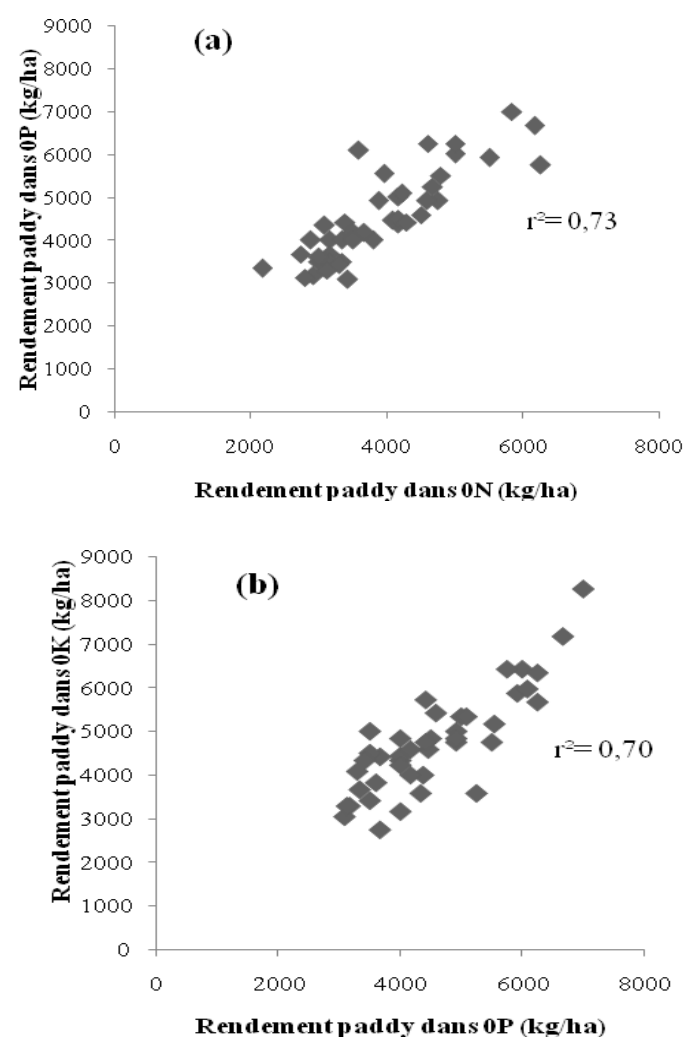

Figure 2 : Relations entre les rendements paddy des parcelles $0 \mathrm{~N}$ et $\mathrm{OP}$ (a) $\mathrm{OP}$ et $0 \mathrm{~K}$ (b) dans la plaine de Bagré. 

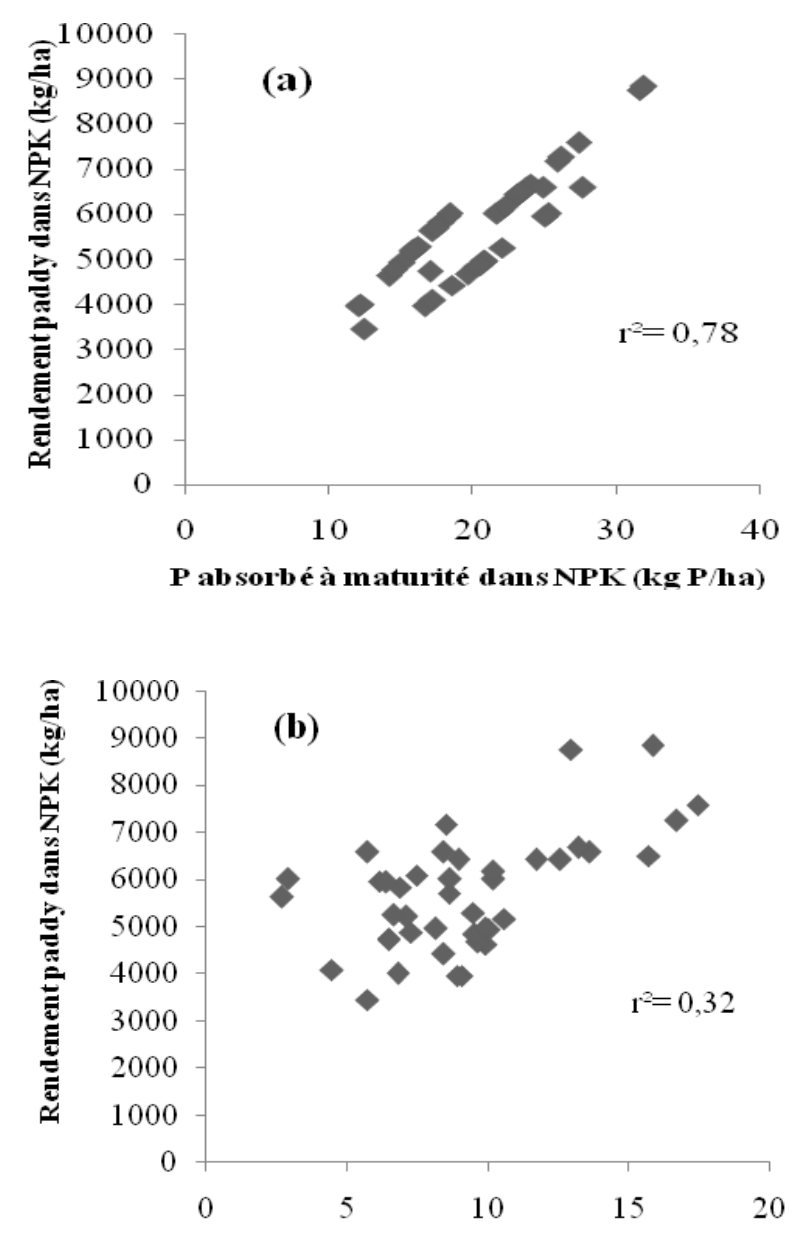

$P$ ab sorb é à maturité dans 0 NOP0K (kg P/ha)

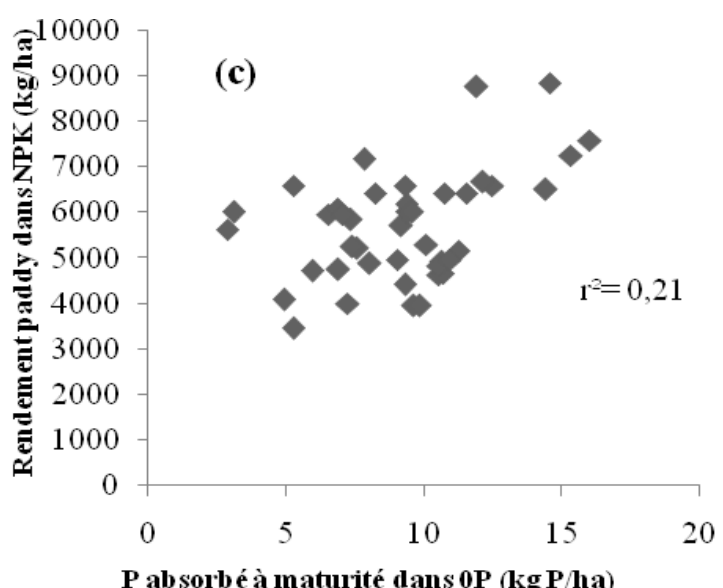

Figure 3: Relations entre le rendement paddy (dans les parcelles avec NPK) et le phosphore absorbé à maturité dans les parcelles avec NPK. (a) dans les parcelles 0NOP0K (b) ou dans les parcelles 0P (c) dans les essais soustractifs conduits en milieu paysan à Bagré, Burkina Faso. Les valeurs représentent les moyennes de trois (3) campagnes successives $(n=42)$. 

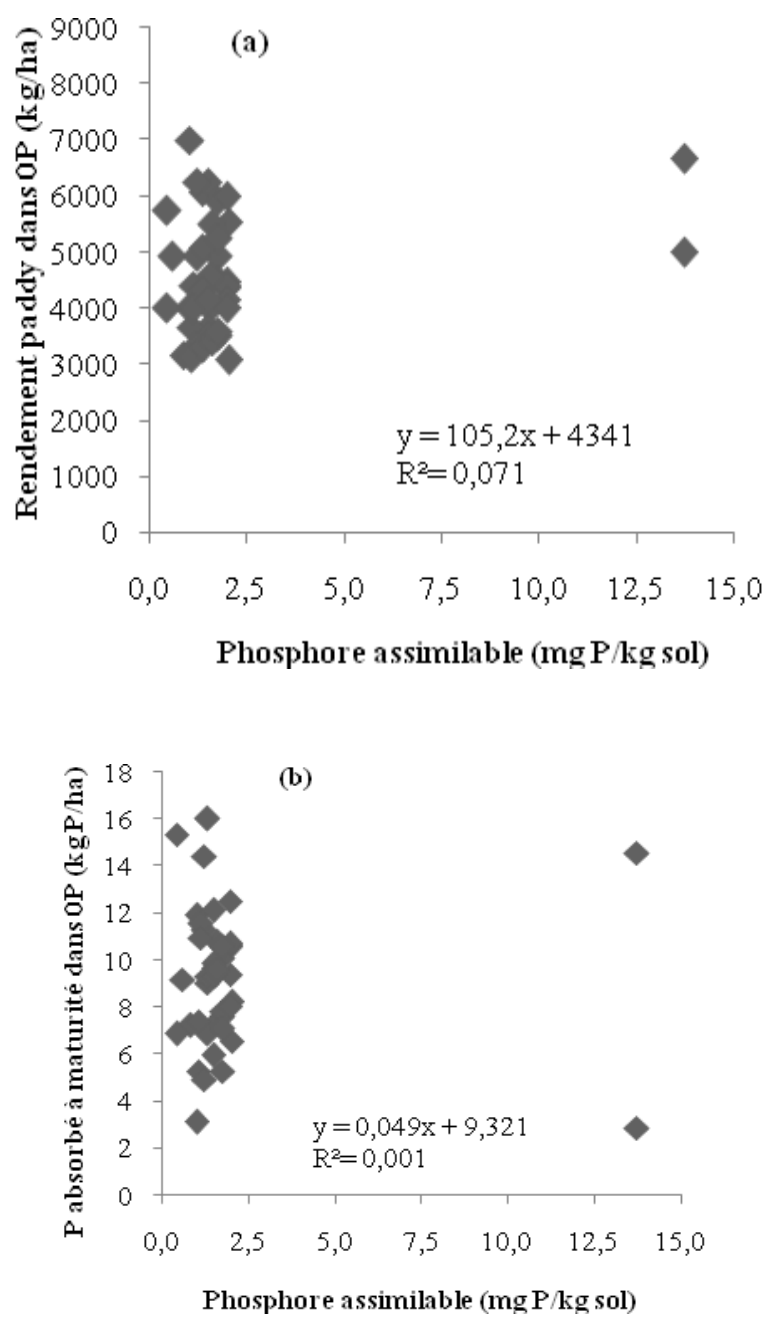

Figure 4 : Relations entre le phosphore assimilable et le rendement paddy (a) et entre le phosphore assimilable et l'accumulation du P (b) dans les traitements $0 \mathrm{P}$.

Tableau 1 : Indicateurs statistiques des résultats d'analyse de sols à Bagré pour le pH eau.

\begin{tabular}{lccccc}
\hline $\begin{array}{l}\text { Variable } \\
\text { Caractéristiques chimiques sols }\end{array}$ & Saison & Moyenne & Minimum & Maximum & Ecart type \\
\hline $\mathrm{pH}$ eau & - & 6,54 & 5,54 & 7,94 & 0,52 \\
$\mathrm{P}$ total $\left(\mathrm{mg} \mathrm{kg}^{-1} \mathrm{sol}\right)$ & - & 137 & 53 & 461 & 84 \\
$\mathrm{P}$ ass. $\left(\mathrm{mg} \mathrm{kg}^{-1} \mathrm{sol}\right)$ & - & 3,75 & 0,43 & 13,74 & 3,31 \\
\hline Capacité nutritive du sol en P & HIV 2003 & 10 & 7 & 13 & 2 \\
CPS (kg P ha & & & & & \\
& CSC 2004 & 12 & 7 & 16 & 3 \\
& HIV 2004 & 11 & 7 & 16 & 2 \\
& Toutes les & & & & 2 \\
& saisons & 11 & 7 & 16 & 2
\end{tabular}

Le phosphore total (P total) et le phosphore assimilable (P ass.) et capacité nutritive du sol en phosphore (CPS) au cours des campagnes HIV 2003, CSC 2004 et HIV 2004 à Bagré, Burkina Faso (n=40). 
Tableau 2 : Réponse du riz au phosphore et efficacité d'utilisation de P dans la plaine rizicole de Bagré (Burkina Faso) au cours de trois campagnes successives.

\begin{tabular}{|c|c|c|c|c|c|}
\hline \multirow[t]{2}{*}{ Saison } & \multicolumn{2}{|c|}{ Augmentation de rendement due à $\mathrm{P}^{1}$} & \multirow{2}{*}{$\begin{array}{l}\text { Efficacité interne de } P^{2} \\
\text { kg grain / kg P absorbé }\end{array}$} & \multirow{2}{*}{$\begin{array}{c}\text { Efficacité agronomique de } P^{3} \\
\Delta \text { kg grain } / \mathrm{kg} P \text { appliqué }\end{array}$} & \multirow{2}{*}{$\begin{array}{c}\text { Variation du P assimilable }^{4} \\
\mathrm{mg} \mathrm{kg}^{-1} \text { sol }\end{array}$} \\
\hline & $\mathrm{kg} \mathrm{ha}^{-1}$ & $\%$ & & & \\
\hline HIV 2003 & $750(559)$ & 14,24 & 290 & $22,2(7,39-59,1)$ & 0,027 \\
\hline CSC 2004 & $1294(822)$ & 19,03 & 246 & $37,4(-6,40-98,5)$ & 0,035 \\
\hline HIV 2004 & $1342(803)$ & 25,28 & 211 & $39,7(13,6-76,4)$ & 0,070 \\
\hline Toutes les saisons & $1139(774)$ & 19,33 & 249 & $33,7(-6,40-98,5)$ & 0,042 \\
\hline
\end{tabular}

1 =Rendement paddy (Ypad) dans les traitements NPK moins le rendement paddy dans les traitements 0P. Les chiffres entre parenthèse représentent

l'écart type par rapport à la moyenne ; 2 =Efficacité interne de $\mathrm{P}=\mathrm{Ypad} / \mathrm{P}$ total absorbé (mesurés en $\mathrm{kg} \mathrm{ha}^{-1}$ ) ; 3 =Efficacité agronomique de

$\mathrm{P}=\left(\mathrm{Ypad}_{\mathrm{NPK}}-\mathrm{Ypad}_{\mathrm{NK}}\right) / \mathrm{P}$ engrais appliqué $\left(\right.$ mesurés en $\left.\mathrm{kg} \mathrm{ha}^{-1}\right)$. Les chiffres entre parenthèse représentent les minima et maxima $; 4=\mathrm{P}$ assimilable dans

les traitements NPK moins P assimilable dans les traitements témoin, tous mesurés en HIV 2004.

Tableau 3 : Concentration de $\mathrm{P}$ dans les grains et la paille $(\% \mathrm{P})$ à la maturité physiologique du riz à Bagré, Burkina Faso.

\begin{tabular}{lcccc}
\hline & Traitement & Moyenne & Minimum & Maximum \\
\hline Grain & Témoin & 0,24 & 0,21 & 0,26 \\
(\% P) & OK & 0,31 & 0,28 & 0,36 \\
& OP & 0,25 & 0,24 & 0,26 \\
ON & 0,30 & 0,28 & 0,33 \\
& NPK & 0,32 & 0,25 & 0,36 \\
& Ensemble & 0,28 & 0,21 & 0,36 \\
\hline Paille & Témoin & 0,08 & 0,08 & 0,09 \\
(\% P) & OK & 0,13 & 0,11 & 0,14 \\
& OP & 0,08 & 0,06 & 0,11 \\
& ON & 0,11 & 0,09 & 0,11 \\
& NPK & 0,09 & 0,07 & 0,11 \\
& Ensemble & 0,10 & 0,06 & 0,14 \\
\hline
\end{tabular}

Les données statistiques sommaires présentées sont basées sur les cultures de trois campagnes successives

de riz en HIV 2003, en CSC 2004 et en HIV $2004(n=42)$. 
Tableau 4 : Bilan partiel du P dans les traitements: témoin, OP et NPK pour trois (3) campagnes successives (HIV 2003, CSC 2004, HIV 2004) dans les parcelles de riz irrigué en milieu paysan à Bagré, Burkina Faso.

\begin{tabular}{|c|c|c|c|c|c|c|}
\hline & Traitement & $\begin{array}{c}P \\
\text { assimilable }\end{array}$ & $\begin{array}{c}\text { Rendement } \\
\text { paddy }\end{array}$ & $\begin{array}{c}P \text { apporté } \\
\text { par les } \\
\text { engrais }\end{array}$ & $\begin{array}{l}\text { P total } \\
\text { absorbé }^{1}\end{array}$ & $\begin{array}{c}\text { Bilan } \\
\text { partiel du } \\
\mathbf{P}^{2}\end{array}$ \\
\hline Saisons & & $\mathrm{mg} \mathrm{kg}^{-1}$ sol & kg ha $^{-1}$ & \multicolumn{3}{|c|}{.................kg $\mathrm{Pha}^{-1} \ldots \ldots \ldots \ldots \ldots$} \\
\hline \multirow[t]{3}{*}{ HIV 2003} & Témoin & 0,146 & 2875 & 0 & 7 & -7 \\
\hline & OP & 0,156 & 4407 & 0 & 8 & -8 \\
\hline & NPK & 0,173 & 5157 & 30 & 16 & +14 \\
\hline \multirow[t]{3}{*}{ CSC 2004} & Témoin & 0,168 & 3798 & 0 & 11 & -11 \\
\hline & OP & 0,155 & 5270 & 0 & 10 & -10 \\
\hline & NPK & 0,204 & 6564 & 30 & 24 & +6 \\
\hline \multirow[t]{3}{*}{ HIV 2004} & Témoin & 0,168 & 2706 & 0 & 8 & -8 \\
\hline & OP & 0,186 & 3703 & 0 & 9 & -9 \\
\hline & NPK & 0,237 & 5044 & 30 & 21 & +9 \\
\hline \multicolumn{7}{|c|}{ Toutes les saisons } \\
\hline \multirow[t]{2}{*}{ Témoin } & Moyenne $^{3}$ & 0,16 & 3200 & 0 & 9 & -9 \\
\hline & Ecart type & 0,01 & 1120 & & & \\
\hline \multirow[t]{2}{*}{ NP $(0 K)$} & Moyenne & 0,22 & 4736 & 30 & 18 & +12 \\
\hline & Ecart type & 0,02 & 1156 & & & \\
\hline \multirow[t]{2}{*}{ NK (OP) } & Moyenne & 0,17 & 4555 & 0 & 9 & -9 \\
\hline & Ecart type & 0,01 & 1053 & & & \\
\hline \multirow[t]{2}{*}{ PK $(0 N)$} & Moyenne & 0,20 & 3918 & 30 & 14 & +16 \\
\hline & Ecart type & 0,01 & 955 & & & \\
\hline \multirow[t]{2}{*}{ NPK } & Moyenne & 0,20 & 5694 & 30 & 21 & +9 \\
\hline & Ecart type & 0,03 & 1208 & & & \\
\hline
\end{tabular}

$1=\mathrm{P}$ total absorbé $=\mathrm{P}$ grain $+\mathrm{P}$ paille.

2 =Bilan partiel net du $\mathrm{P}=\mathrm{P}$ apporté par les engrais $-\mathrm{P}$ absorbé (grains + paille).

3 =Moyenne et écart type de trois campagnes.

\section{DISCUSSION}

Analyse de sols et capacité nutritive du sol en phosphore

Les valeurs du pH_eau ont été faiblement acides à neutre, ce qui n'a pas influencé l'absorption des éléments minéraux par le riz. Kirk et al. (2003) ont obtenu des résultats similaires en sols inondés. Les valeurs du phosphore assimilable sont faibles (3,75 $\left.\mathrm{mg} \mathrm{P} \mathrm{kg} \mathrm{sol}^{-1}\right)$, valeurs en dessous des niveaux relevés dans la littérature (Haefele, 2001). Cet élément constitue avec l'azote et le potassium l'un des principaux éléments nutritifs dont le riz a besoin pour son développement et cela a été prouvé par plusieurs essais de fertilisation dans la région soudano sahélienne. Haefele et al. (2001) ont indiqué que dans la Vallée du Fleuve Sénégal, le phosphore assimilable devenait un facteur limitant pour le riz à des valeurs de P-Bray1 en dessous de $4 \mathrm{mg} \mathrm{kg}^{-1}$ sol. Cette carence en phosphore pourrait contribuer à une réduction induite du taux de recouvrement de l'azote par la plante.

Réponse du riz à l'application du phosphore et efficacité d'utilisation de $\mathbf{P}$ Réponse du riz à l'application de phosphore

Le phosphore a été le second facteur limitant des rendements après l'azote sur le périmètre de Bagré ce qui est expliqué par les rendements bas obtenus respectivement avec les traitements sans apport de $\mathrm{N}$ et sans apport de P. Plusieurs auteurs ont obtenu des 
résultats similaires en Asie (Dobermann et al., 2002; Dobermann et al., 2003a) et en Afrique soudano-sahélienne (Haefele, 2001). L'augmentation de rendement due à l'application des éléments nutritifs a été de $19 \%$ pour le phosphore. La réponse observée du phosphore a reflété l'effet des engrais appliqués et celui provenant du sol comme l'ont mis en évidence Haefele et al. (2003).

\section{Efficacité d'utilisation du phosphore}

Dans les conditions optimales de nutrition et de faibles contraintes à la croissance, l'efficacité interne du phosphore devrait se situer autour de $385 \mathrm{~kg}$ paddy $\mathrm{kg}^{-1} \mathrm{P}$ absorbé (Dobermann and Fairhurst, 2000). Les résultats très variables qui ont été obtenus au regard de cet indicateur ont montré que certaines contraintes ont probablement influencé négativement (pour les faibles valeurs) la nutrition phosphorique du riz. Par contre, les fortes valeurs de EiP ont confirmé l'hypothèse d'une meilleure gestion du riz et une meilleure efficacité d'utilisation du phosphore. Dobermann et al. (2003a) ont observé en Asie des corrélations fortes entre les rendements grains des parcelles $0 \mathrm{~N}, 0 \mathrm{P}$ et $0 \mathrm{~K}$. Les fortes corrélations entre les rendements grains des différents traitements reflètent les variations inhérentes de la capacité nutritive du sol et du climat. Elles reflètent également les différences entre les parcelles dues à la gestion des cultures (Dobermann et al., 2003b). La relation entre les analyses de sols et le phosphore absorbé dans les plaines rizicoles est fortement affectée par la teneur en argile, la minéralogie de l'argile, la saturation en bases, les conditions d'oxydo-réduction. Ces conditions entraînent de grandes variabilités pour les valeurs critiques reportées dans la littérature (Haefele, 2001; Haefele et al., 2003). Bien que la variabilité de la capacité nutritive du sol en phosphore soit très grande, cet élément nutritif est moins limitant au niveau actuel des rendements. Il est également moins affecté par les variations intra saisonnières par rapport à la capacité nutritive du sol en azote. Il peut aussi être manipulé plus facilement à travers une gestion de l'ensemble du bilan des entrées-sorties parce que les pertes de phosphore dans les plaines rizicoles sont en général faibles (Haefele, 2001; Haefele et al., 2003).

\section{Bilan partiel du $\mathbf{P}$}

L'application de $\mathrm{P}$ a permis de compenser les pertes dues à l'exportation par les cultures. En effet, il a été démontré en rive droite que le riz répondait assez bien à l'application de phosphore (Segda, 2001). Le phosphore peut être apporté par l'eau d'irrigation ou de pluie. La réponse observée du $\mathrm{P}$ reflète l'effet des engrais appliqués et celui provenant du sol (Haefele, 2001; Haefele et al., 2003). La dynamique de $\mathrm{P}$ dans les sols submergés est particulièrement complexe. En effet, le $\mathrm{P}$ inorganique du sol se trouve dans une très large mesure adsorbé, ou co-précipité, sur les oxydes de fer (Fe) et d'aluminium (Hinsinger, 2001). La réduction du $\mathrm{Fe}(\mathrm{III})$ et donc leur dissolution en $\mathrm{Fe}(\mathrm{II})$, entraîne une libération de $\mathrm{P}$ et, de fait, une augmentation de la biodisponibilité de $\mathrm{P}$ suite à l'inondation des rizières (Liesack et al., 2000). Cependant, en sol acide, comme l'abaissement du potentiel redox s'accompagne d'une hausse de $\mathrm{pH}$, le $\mathrm{Fe}(\mathrm{II})$ devient rapidement moins soluble et forme des précipités qui immobilisent P. Contrairement à l'azote, la maximisation de l'efficacité de recouvrement de $\mathrm{P} \_$engrais appliqué est moins importante que la prédiction des besoins en $\mathrm{P}$ et de la quantité à apporter pour atteindre un rendement ciblé et maintenir les réserves en $\mathrm{P}$ (Haefele, 2001). Cela se conçoit dans la mesure où $\mathrm{P}$ est moins mobile dans le sol, les apports exogènes de $\mathrm{P}$ autres que les engrais sont faibles et une part importante de $\mathrm{P}$ engrais résiduel restant dans le sol peut être disponible pour les campagnes rizicoles suivantes. Des apports de 20-25 kg P ha ${ }^{-1}$ peuvent permettre de maintenir des rendements de 5-6 $\mathrm{t} \mathrm{ha}^{-1}$. Si l'on veut atteindre des rendements de 7-8 $\mathrm{t} \mathrm{ha}^{-1}$, il faut en ce moment appliquer d'autres stratégies de gestion à long terme (Dobermann and Fairhurst, 2000). Comme souligné par Haefele (2001), une telle stratégie doit se baser sur la connaissance des besoins phosphatés du riz en fonction de la disponibilité des autres 
éléments nutritifs, la connaissance du statut phosphaté du sol, la connaissance du bilan phosphorique qui tiendra compte du P exporté par les récoltes, $\mathrm{P}$ recyclé par les résidus et les apports par les engrais et la connaissance des processus qui gouvernent les effets résiduels de P_engrais, afin de prévoir la biodisponibilité du P résiduel pour les cultures subséquentes.

\section{Conclusion}

La caractérisation chimique de la fertilité des sols du périmètre irrigué de Bagré indique des valeurs du $\mathrm{pH}$ se situant dans des plages favorables au développement du riz. Le périmètre présente de très faibles teneurs en phosphore total et assimilable qui ne permettent pas une alimentation optimale des cultures. La capacité nutritive en phosphore des sols est relativement faible. L'apport de phosphore améliore très sensiblement l'efficacité physiologique interne du phosphore. La majeure partie du phosphore total absorbé est contenue dans les grains. Le bilan minéral moyen du phosphore est positif avec l'apport de cet élément. Dans les conditions agro-pédologiques de la plaine rizicole de Bagré, l'apport de $\mathrm{P}_{-}$engrais améliore le rendement du riz paddy ainsi que l'efficacité agronomique et interne.

\section{REMERCIEMENTS}

Nous remercions les producteurs de Bagré au Burkina Faso. Cette étude a été financée par le Centre International pour la Fertilité des sols et le Développement agricole (IFDC) Division Afrique à travers le Fonds International de Développement Agricole (FIDA), et par l'Institut de l'Environnement et de Recherches Agricoles (INERA) du Burkina Faso.

\section{REFERENCES}

BEGE. 2008. Integrated feasibility study on the biomass in the valleys of Sourou and Bagré for ethanol and electricity productions. Final consultation report. Ministry of Mines and Energy, Ouagadougou, Burkina Faso, p. 183.
De Vries ME, Leffelaar PA, Sakané N, Bado BV, Giller KE. 2011. Adaptability of irrigated rice to temperature change in Sahelianenvironnements. Exp. Agric., 47: 69-87.

Dobermann A, Fairhurst TH. 2000. Rice: Nutritional Disorders and Nutrient Management. International Rice Research Institute \& Potash and Phosphate Institute: Singapore; p. 191.

Dobermann A, Witt C, Abdulrachman S, Gines HC, Nagarajan R, Son TT, Tan PS, Wang GH, Chien NV, Thoa VTK, Phung CV, Stalin P, Muthukrishnan P, Ravi V, Babu M, Simbahan GC, Adviento MAA. 2003a. Soil fertility and indigenous nutrient supply in irrigated rice domains of Asia. Agron. J., 95(4): 913-923.

Dobermann A, Witt C, Abdulrachman S, Gines HC, Nagarajan R, Son TT, Tan PS, Wang GH, Chien NV, Thoa VTK, Phung CV, Stalin P, Muthukrishnan P, Ravi V, Babu M, Simbahan GC, Adviento MAA, Bartolome V. 2003b. Estimating indigenous nutrient supplies for sitespecific nutrient management in irrigated rice. Agron. J., 95(4): 924-935.

Dobermann A, Witt C, Dawe D, Gines GC, Nagarajan R, Satawathananont S, Son TT, Tan PS, Wang GH, Chien NV, Thoa VTK, Phung CV, Stalin P, Muthukrishnan P, Ravi V, Babu M, Chatuporn S, Kongchum M, Sun Q, Fu R, Simbahan GC, Adviento MAA. 2002. Site-specific nutrient management for intensive rice cropping systems in Asia. Field Crops Res., 74: 3766.

FAO. 2003. Assessment of Soil Nutrient Balance. Approaches and Methodologies, Roy RN, Misra RV, Lesschen JP. Smaling EM (eds). FAO Fertilizer \& Plant Nutrition Bulletin $\mathrm{N}^{\circ}$ 14: Rome.

Haefele SM. 2001. Improved and sustainable nutrient management for irrigated ricebased cropping systems in West Africa. $\mathrm{PhD}$ thesis, Hamburger Bodenkundliche Arbeiten; p. 241.

Haefele SM, Wopereis MCS, Donovan C. 2002. Farmers' perceptions, practices and 
performance in a Sahelian irrigated rice scheme. Expl. Agric., 38: 197-210.

Haefele SM, Wopereis MCS, Donovan C, Maubuisson J. 2001. Improving the productivity and profitability of irrigated rice production in Mauritania. Eur. J. Agron., 14(3): 181-196.

Haefele SM, Wopereis MCS, Ndiaye MK, Barro SE, OuldIsselmou M. 2003. Internal nutrient efficiencies, fertilizer recovery rates and indigenous nutrient supply of irrigated lowland rice in Sahelian West Africa. Field Crops Res., 80(1): 19-32.

Hinsinger H. 2001. Bio-availability of soil inorganic $\mathrm{P}$ in the rhizosphere as affected by root-induced chemical changes: a review. Plant Soil., 237: 173-195.

INERA-DGPER. 2010. Renforcement de la Disponibilité et de l'Accès aux Statistiques Rizicoles : Une contribution à l'initiative d'urgence pour le Riz en Afrique Subsaharienne. Rapport pays : Burkina Faso. AfricaRice, Ouagadougou, 2010; p. 75.

Kanté S. 2001.Gestion de la fertilité des sols par classe d'exploitation au Mali-Sud. PhD Thesis, Wageningen University, The Netherlands; p. 236.

Kirk GJD, Solivas JL, Alberto MC. 2003. Effects of flooding and redox conditions on solute diffusion in soil. European Journal of Soil Science., 54:617-624.

Lacharme M. 2001. Mémento Technique de Riziculture pour la Vallée du Fleuve Sénégal, du Développement Rural et de l'Environnement. Direction de la Recherche Formation Vulgarisation : Saint-Louis, Sénégal; 168.

Liesack W, Schnell S, Revsbech NP. 2000. Microbiology of flooded rice paddies. FEMS Microbiology Reviews., 24: 625645.

Roy RN, Finck A, Blair GJ, Tandon HLS. 2006. Plant Nutrition for Food Security: a Guide for Integrated Nutrient Management. FAO Fertilizer and Plant Nutrition Bulletins, 16, FAO 107288: Rome; 237-239.
Segda Z. 2001. Analyse des contraintes liées au sol, aux pratiques culturales et à la gestion de l'azote en riziculture irriguée. Cas du village de Nimatoulaye dans la plaine de Bagré. Mémoire de DEA (Diplôme d'Etudes Approfondies) en "Sciences Biologiques Appliquées", Option "Biologie et Ecologie Végétales", UFR-SVT, Université de Ouagadougou; p. 53.

Sims JT. 2000. Soil test phosphorus: Olsen P. In Methods of Phosphorous Analysis for Soils, Sediments, Residuals, and Waters, Pierzynski GM (Ed). Southern Cooperative Series Bulletin no. 396; NC North Carolina State University: North Carolina State; 20-21.

Van Asten PJA, Wopereis MCS, Haefele SM, Ould Isselmou M, Kropff MJ. 2003. Explaining yield gaps on farmer-identified degraded and non-degraded soils in a Sahelian irrigated rice scheme. NJASWageningen Journal of Life Sciences, 50(3-4): 277-296.

Van Reeuwijk LP. 2002. Procedures for Soil Analysis $\quad\left(6^{\text {th }}\right.$ edn). ISRIC-FAO: Wageningen; 120.

Wang W, Chris JS, Chalk PM, Chen D. 2001. Evaluating Chemical and Physical Indices of Nitrogen Mineralization Capacity with an Unequivocal Reference. Soil Sc. Soc. Am. J., 65: 368-376.

Witt C. 2003. Fertilizer Use Efficiencies in Irrigated Rice in Asia. IFA regional conference for Asia and the Pacific: Cheju Island, Republic of Korea; 13.

Wopereis MCS, Haefele SM, Kebbeh M, Miezan K, Diack BS. 2001. Improving the productivity and profitability of irrigated rice production in the Sahelian West Africa. In: Proceedings of the Expert Consultation Meeting on Yield Gap and productivity decline in rice production at FAO, Rome, Italy, 5-7 september 2000. 